\title{
Theory of Frequency Comb Generation in Cavity Enhanced Second Harmonic Generation
}

\author{
François Leo, ${ }^{1 *}$ Tobias Hansson, ${ }^{2,3}$ Iolanda Ricciardi, ${ }^{4}$ Maurizio De Rosa, ${ }^{4}$ \\ Stéphane Coen, ${ }^{1}$ Stefan Wabnitz, ${ }^{3,4}$ and Miro Erkintalo ${ }^{1}$ \\ ${ }^{1}$ The Dodd-Walls Centre for Photonic and Quantum Technologies, Department of Physics, The University of \\ Auckland, Auckland 1142, New Zealand \\ ${ }^{2}$ Department of Applied Physics, Chalmers University of Technology, SE-41296 Göteborg, Sweden \\ ${ }^{3}$ Dipartimento di Ingegneria dell'Informazione, Università di Brescia, via Branze 38, 25123 Brescia, Italy \\ ${ }^{4}$ CNR-INO, Istituto Nazionale di Ottica, Via Campi Flegrei 34, 80078 Pozzuoli (NA), Italy \\ ${ }^{*}$ Corresponding author:f.leo@auckland.ac.nz
}

\begin{abstract}
We theoretically investigate frequency comb generation in dispersive quadratically nonlinear resonators. We introduce a single mean field equation to model cavity enhanced second harmonic generation and find excellent agreement with recent experimental frequency comb observations.
\end{abstract}

OCIS codes: (190.4410) Nonlinear optics, parametric processes, (190.3100) Instabilities and chaos

Generation of frequency combs from microresonators has attracted significant attention since the first experimental demonstration in a silica toroid almost a decade ago [1]. The physical mechanisms underlying such broadband frequency conversion is now very well understood and has been linked to temporal dissipative structures previously studied in fibre cavities [2,3]. These combs arise through the interplay between group velocity dispersion (GVD) and cubic (Kerr) nonlinearity. Interestingly, recent results in a bulk resonator demonstrated the possibility of generating frequency combs from a purely quadratic nonlinear resonator [4]. This observation, combined with the recent development of quadratically nonlinear microresonators [5], suggests that a novel way of generating integrated frequency combs might be possible. However, no modelling of this process has been put forward and the underlying physics is still poorly understood. Here we introduce a mean field equation that allows for the modelling of the dynamics of cavity enhanced second harmonic generation (CSHG) [6]. We mainly focus on the case of singly resonant CSHG as experimental data is readily available for comparison [4]. Nevertheless, we have found that the same equation (with a different nonlinear response function) also permits investigating doubly resonant CSHG.

We start from the well known propagation equations describing dispersive second harmonic generation [7]. In the singly resonant configuration, the second harmonic $(\mathrm{SH})$ wave is removed at the end of each roundtrip, slaving it to the fundamental. This boundary condition allows approximately solving the $\mathrm{SH}$ propagation equation [8] and turn the system into a single equation for the fundamental, with a delayed nonlinear response. In the condition of low loss for the fundamental field, that equation can in turn be averaged into a mean field equation that describes the evolution of the intracavity field. It reads:

$$
t_{\mathrm{R}} \frac{\partial A(t, \tau)}{\partial t}=\left[-\alpha_{1}-i \delta_{1}-i L \frac{k_{1}^{\prime \prime}}{2} \frac{\partial^{2}}{\partial \tau^{2}}\right] A-\rho A^{*}\left[A^{2}(t, \tau) \otimes I(\tau)\right]+\sqrt{\theta_{1}} A_{\text {in }} .
$$

Here, $t$ is the "slow time" variable linked to the roundtrip index and $\tau$ is the "fast time" [2], $t_{\mathrm{R}}$ is the cavity roundtrip time, $A(t, \tau)$ is the fundamental field (expressed in $\sqrt{\mathrm{W}}$ ), $\alpha_{1}$ are the total intracavity losses, $\delta_{1}$ is the phase detuning from the closest cavity resonance, $k_{1}^{\prime \prime}$ is the GVD at the fundamental, $L$ is the cavity length, $\rho=(\kappa L)^{2}$ where $\kappa$ is the effective quadratic nonlinear coefficient, $\otimes$ denotes convolution, the nonlinear response function $I(\tau)$ describes the interaction with the SH wave, $\theta_{1}$ is the coupling ratio of the input coupler and $A_{\text {in }}$ is the pump field. The delayed response $I(\tau)$ is defined as $I(\tau)=\mathscr{F}^{-1}[\hat{I}(\Omega)]$ where $\hat{I}(\Omega)=\left[\left(1-e^{-i x}-i x\right) / x^{2}\right], x(\Omega)=[\Delta k+i \hat{k}(\Omega)] L, \Delta k$ denotes the phase mismatch, $\hat{k}(\Omega)=-\alpha_{c, 2} / 2+i\left[\Delta k^{\prime} \Omega+\left(k_{2}^{\prime \prime} / 2\right) \Omega^{2}\right], \alpha_{c, 2}$ are the propagation losses around the SH, $\Delta k^{\prime}$ is the group velocity mismatch between the fundamental and the SH and $k_{2}^{\prime \prime}$ corresponds to the GVD at the SH wavelength. See [6] for more details.

To validate our model, we integrate Eq. (1) with the parameters corresponding to the phase-matched CSHG experiments in [4]. We find excellent agreement with the experimental results as can be seen in Fig. 1. The combs seem to originate from a modulation instability (MI)-like growth of sidebands around the fundamental. Interestingly, previous 
investigations in spatially diffractive quadratic resonators have shown that MI cannot occur unless the SH process is highly phase mismatched [9]; here, in contrast, we are observing MI for the case of perfect phase-matching. The main difference between the temporal case and the spatial analog is that the spatial walk-off (physically corresponding to a mismatch of the two Poynting vectors) is small and often neglected. This indicates that the temporal walk-off plays a similar role as the phase mismatch in destabilizing the $\mathrm{CW}$ field. To gain further insight into this process, and in
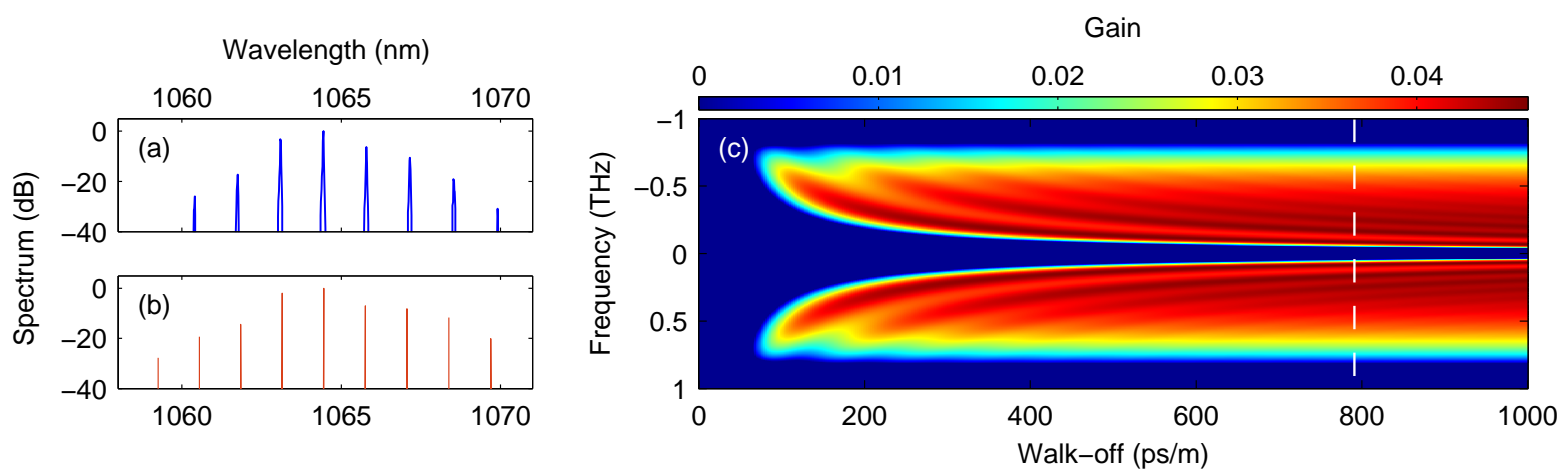

Fig. 1. Frequency comb generation from a CSHG system. (a) Experiment [4] with $2 \mathrm{~W}$ input power (b) Simulation with $2 \mathrm{~W}$ input power $\left(\left|A_{\text {in }}\right|^{2}\right), \delta_{1}=-0.02$. (c) MI gain as a function of frequency and walk-off. The white line highlights the walk-off value corresponding to Fig.1 (a),(b).

particular to investigate the role of the walk-off, we perform a linear stability analysis of Eq. (1). The MI gain for different walk-off values is presented in Fig. 1 (c). The walk-off corresponding to Figs. 1 (a) and (b) is indicated by the dashed white line. The crucial role of the walk-off is highlighted by the fact that, as known from earlier spatial studies [9], there can be no MI in the absence of walk-off. This is due to the important role played by the nonlinear losses on the MI gain. The MI indeed originates from the interplay between three-wave-mixing gain (corresponding to an optical parametric oscillator with the SH as a pump) and a competing sum frequency generation (SFG) process that acts as a nonlinear loss. The latter is proportional to the squared sinc-function describing the SFG efficiency that is constant and highly efficient in the absence of phase mismatch and walk-off (the GVD of the SH plays a negligible role). Hence MI can only arise when the SFG is phase mismatched through strong walk-off [6].

We stress that Eq. (1) was derived by considering that the SH wave is removed after each roundtrip. Although relevant to bulk cavities, this is different from what would happen in a high finesse microresonator [5], where both the fundamental and the SH fields are expected to resonate simultaneously. We have also analyzed such doubly-resonant CSHG, and found a similar kind of walk-off induced MI as in the singly-resonant case discussed above. Significantly, we have found that, barring a small additional approximation, doubly-resonant CSHG can be reduced to an equation formally identical with Eq. (1). The only difference being the response function $I(\tau)$, which is highly impacted by the resonance around the $\mathrm{SH}$.

In conclusion, we have derived a new single mean field equation that fully captures the temporal and spectral dynamics in singly and doubly resonant CSHG. We obtain excellent agreement with recent experimental results and our analysis sheds new light on the physical origin of the MI process. We believe this walk-off induced modulation instability is likely to appear in many different configurations of quadratic resonators.

\section{References}

1. P. Del'Haye, A. Schliesser, O. Arcizet, T. Wilken, R. Holzwarth, and T. J. Kippenberg, Nature 450, 1214-1217 (2007).

2. S. Coen, H. G. Randle, T. Sylvestre, and M. Erkintalo, Opt. Lett. 38, 37 (2013).

3. F. Leo, S. Coen, P. Kockaert, S.-P. Gorza, Ph. Emplit, and M. Haelterman, Nature Photon. 4, 471 (2010).

4. I. Ricciardi, S. Mosca, M. Parisi, P. Maddaloni, L. Santamaria, P. De Natale, and M. De Rosa, Phys. Rev. A 91, 063839 (2015).

5. P. S. Kuo, J. Bravo-Abad, and G. S. Solomon, Nat Commun 5, 3109 (2014).

6. F. Leo, T. Hansson, I. Ricciardi, M. De Rosa, S. Coen, S. Wabnitz, and M. Erkintalo, Phys. Rev. Lett. 116, 033901 (2016).

7. C. R. Menyuk, R. Schiek, and L. Torner, J. Opt. Soc. Am. B 11, 2434 (1994).

8. B. Zhou, H. Guo, and M. Bache, Phys. Rev. A 90, 013823 (2014).

9. P. Lodahl and M. Saffman, Opt. Commun. 184, 493 (2000). 